Proceedings of the 1999 IEEE

International Conference on Robotics \& Automation

Detroit, Michigan - May 1999

\title{
Examples of 3D Grasp Quality Computations
}

\author{
Andrew T. Miller Peter K. Allen \\ Department of Computer Science, Columbia University, New York, NY $10027^{*}$
}

\begin{abstract}
Previous grasp quality research is mainly theoretical, and has assumed that contact types and positions are given, in order to preserve the generality of the proposed quality measures. The example results provided by these works either ignore hand geometry and kinematics entirely or involve only the simplest of grippers. We present a unique grasp analysis system that, when given a $3 D$ object, hand, and pose for the hand, can accurately determine the types of contacts that will occur between the links of the hand and the object, and compute two measures of quality for the grasp. Using models of two articulated robotic hands, we analyze several grasps of a polyhedral model of a telephone handset, and we use a novel technique to visualize the $6 D$ space used in these computations. In addition, we demonstrate the possibility of using this system for synthesizing high quality grasps by performing a search over a subset of possible hand configurations.
\end{abstract}

\section{Introduction}

Choosing a good grasp requires some method of evaluating a grasp. The current methods are largely theoretical and have the stated goal of finding the optimum placement of contacts on an object. This allows the various grasp quality metrics that are proposed to be as general as possible. However, by analyzing a particular contact placement rather than a hand configuration, the metrics effectively ignore the geometry and kinematics of the particular hand being used to create the grasp. It is possible that the optimum placement of contacts on an object is not reachable by any physical hand.

The field of grasping still lacks a general system which can apply these theories to evaluate the grasps formed by actual hands. Such a system would not only be useful as a more realistic tool for grasp planning, but it would also allow us to test a hand's ability to grasp different kinds of objects. This would lead to the

*This work was supported in part by an ONR/DARPA MURI award ONR N00014-95-1-0601, DARPA AASERT awards DAAHO4-93-G-0245 and DAAH04-95-1-0492, and NSF grants CDA-96-25374 and IRI-93-11877. comparison of hand designs to find the one best suited to particular kinds of tasks. Until now, hand designs have emulated the human hand because of its proven ability to perform complex grasping and manipulation tasks, but a system with these capabilities would be an indispensable tool for designing and testing alternate possibilities.

The increasing power of the currently available tools for geometric modeling and computational geometry have made such a system possible. In this report, we present our initial version of a unique tool for general grasp analysis. Given 3D models of a hand and an object, and using the ACIS geometric modeling engine, our system reports contact types and locations with high precision for any configuration of the hand. These contact descriptions are then used to accurately compute the previously proposed quality measures. We have designed the system to be as flexible and realistic as possible, and this is demonstrated by its ability to handle complex contact geometries, frictional forces, and a wide variety of hand kinematics.

Another benefit of our system is its unique method for visualizing the results of its analyses. Previous authors have only provided results obtained from computing the quality of $2 \mathrm{D}$ grasps, because of the difficulty in displaying the $6 \mathrm{D}$ output of $3 \mathrm{D}$ grasp quality computations. However, we have chosen projections of this $6 \mathrm{D}$ space that can convey useful information about the characteristics of a particular grasp, and we provide examples of this when we present our results.

The remainder of this paper is laid out as follows. Section 2 briefly reviews some of the previous research. Section 3 explains the process of computing grasp quality and describes the specifics of our grasp evaluation system. Section 4 presents our current results of analyses performed with models of the Barrett and DLR robotic hands. Section 5 summarizes our contributions and details our proposed research directions.

\section{Related Work}

There is a great deal of previous research in the field of grasp analysis and synthesis. However, due to 
space constraints we can only highlight a few of the most relevant papers. See reviews by Mishra and Silver [8] and by Troccaz [10] for references of some of the older work. Salisbury [12] classified types of contacts, with and without friction, between two bodies and provided an approach that accounts for uni-sense contact wrenches in determining whether a grasp completely restrains an object which he calls form-closure. Pollard [11] developed a parallel system to compute high quality grasps using prototype grasps as input, and Fischer and Hirzinger [5] created a system that repeatedly chooses 3 contact points on an object using a heuristic and checks whether these points can be realized by the hand. Given the goal of minimizing the sum magnitude of the contact forces, Kirkpatrick et al. [6] proposed a general measure of quality for an $n$-contact grasp, defining it as the radius of the largest wrench space ball which just fits within the unit grasp wrench space. Ferrari and Canny [4] developed this measure further and proposed another measure minimizing the maximum contact force. Earlier, $\mathrm{Li}$ and Sastry [7] noted that similar measures are not invariant to the choice of torque origin, and proposed using the volume of the grasp wrench space as an invariant quality measure. They also developed a quality measure using task ellipsoids to better model the space of wrenches required for a task, whereas the previous authors all assumed the task wrench space is unknown and therefore defined the space as a ball centered at the origin of the wrench space. Our overview of the process of computing a grasp's quality most resembles the presentation by Ferrari and Canny.

\section{The Grasping Simulator}

We have built a grasping simulator which when given a model of an object, a model of a hand, and a hand configuration, can compute two quality measures of the resulting grasp and display various projections of the convex hull used in this computation. To accomplish this, it performs four phases: hand construction, contact location, quality computation, and hull projection. We describe each of these phases in the following sections.

\subsection{Hand Construction}

We have designed the simulator to be as general as possible so that it is useful for a variety of hands and objects. However, it was necessary create standard formats for the definitions of a hand and an object, so that they can be understood by the system. Objects are fairly simple and consist only of a CAD model file and a material specification. An object is currently assumed to be composed of a single material of uni- form density. This simplifies the computation of the object's center of mass and the computation of static friction between the surface and links of the hand.

Similarly, our hand description file allows us to accurately describe the link geometries as full $3 \mathrm{D}$ entities, each with an associated material specification. Furthermore, it also describes the kinematics of the hand using standard Denavit-Hartenberg parameters and additional information regarding joint limits and coupled (passive) joints. This general format is flexible enough to describe hands as simple as a parallel jaw gripper or as complicated as many of the fully articulated hands in use today.

After the hand model has been constructed, the simulator reads a hand configuration which sets the hand transform and sets the value of each active joint parameter up to but not including the last active joint in each finger, leaving it to be moved during the next phase.

\subsection{Contact Location}

Since we are currently evaluating grasps for stability, contacts between links and the object can only improve grasp quality, and thus, it is assumed that if a finger can touch an object, it should do so. Accordingly, we use a hand configuration to fix the hand parameters up to the last active joint in each finger chain and determine if the link or links following this joint can contact the object for any value within the legal limits of the joint. If a contact will occur, we search for the joint value that will cause this. By ignoring configurations which do not result in contact, this method allows us to specify a unique hand configuration using one fewer parameter per finger. Unfortunately, a purely analytic closed-form solution to this problem is not possible because of complications introduced by coupled joints and complex link geometries, and currently we have implemented a modified binary search using the geometry system's clearance function which finds the minimum distance between a link and the object.

\subsection{Computing Grasp Quality}

At this point, we can assume that any link that can touch the object in the given configuration does so, and the grasp is ready to be analyzed. First, the system must analyze each contact and determine its contribution to the overall stability of the grasp. Then we compute a convex hull of the contact wrenches to determine the overall space of wrenches that can be applied by this grasp. Using the hull, the system can compute two commonly used measures of grasp quality. We describe each of the steps in the subsections that follow. 


$$
\begin{gathered}
\mathbf{f} \approx \sum_{j=1}^{m} \alpha_{j} \mathbf{f}_{j} \\
\alpha_{j} \geq 0, \sum_{j=1}^{m} \alpha_{j}=1
\end{gathered}
$$

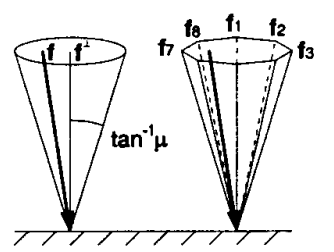

Figure 1: We have approximated the friction cone with an 8 sided pyramid. The contact force, $f$, is represented as a convex combination of 8 force vectors around the boundary of the cone. Note the friction cone is usually drawn on the interior of an object and contact forces point out of the cone. For ease of display in our later figures we have inverted this convention.

\subsubsection{Contact Analysis}

The simulator identifies contacts by intersecting the object with each link including the palm, resulting in a set of common surface patches between the links and the object. Each independent region is examined individually. If the patch consists only of a point, then we classify it as a point contact. If the patch has two vertices then the contact is classified as a line contact, and for a patch with greater than two vertices, we consider it a plane contact. Nguyen [9] points out that these complex contacts (linear and planar) can be represented as the convex sum of proper point contacts at each of the vertices of the surface patch.

To find the direction that forces may be applied at a particular contact, we need to find the local contact normal which is defined as the inward pointing normal of the tangent plane of the contact. If one surface has a defined tangent plane at the point of contact, this is also the contact tangent plane. In the case of a edge touching a non-parallel edge, a tangent plane can be defined by the tangent vectors of each curve. For all other cases, such as a vertex contacting an edge or two vertices contacting, a tangent plane cannot be defined and the contact is considered unstable and is disregarded for the remainder of the process.

Let us assume we have broken up the complex contacts so that we have a collection of $n$ point contacts between the links and the object. We will index each of these contacts later, but for now we examine the forces acting at one particular contact. If we assume a Coulomb friction model, then the total force, $\mathbf{f}$, acting on the object at a contact point must lie within a cone that has an apex at the contact point, an axis along the contact normal, and a half angle of $\tan ^{-1} \mu_{s}$, where $\mu_{s}$ is the coefficient of static friction. In order to find the total grasp wrench space we will need a finite basis set of vectors, so it is necessary to approximate this cone with a proper pyramid (see figure 1).

\subsubsection{Convex Hull Construction}

Given this approximation of the space of forces that can be applied at contact point $i$, the system must now determine the corresponding space of wrenches that can be applied to the object by that contact. To accomplish this, we require a torque multiplier, $\lambda$, that relates units of torque to units of force. For this work, we have chosen to enforce $\|\tau\| \leq\|\mathbf{f}\|$ by choosing $\lambda=$ $\frac{1}{r}$, where $r$ is the maximum radius from the torque origin, often the center of gravity. This will ensure that the quality of a grasp is independent of object scale [11]. Each of the $m$ force vectors representing the boundary of the friction cone is translated to the wrench space origin by computing the corresponding torque such that

$$
\mathbf{w}_{i, j}=\left(\begin{array}{c}
\mathbf{f}_{i, j} \\
\lambda\left(\mathbf{d}_{i} \times \mathbf{f}_{i, j}\right)
\end{array}\right)
$$

where $\mathbf{f}_{i, j}$ is one the $m$ force vectors on the boundary of the friction cone at contact $i$, and $\mathbf{d}_{i}$ is the vector from the torque origin to the $i$-th point of contact. These wrenches form the boundary of the wrenches that can be applied at that contact point given a unit normal force.

Ferrari and Canny [4] describe two methods of finding the unit grasp wrench space. One limits the maximum magnitude of the contact normal forces to 1 , and the other limits their sum magnitude to 1 . We have implemented the second option due to its ease of computation. Under this constraint, the set of wrenches that can be exerted on the object is:

$$
W=\operatorname{ConvexHull}\left(\bigcup_{i=1}^{n}\left\{\mathbf{w}_{i, 1}, \ldots, \mathbf{w}_{i, m}\right\}\right)
$$

If this convex hull contains the wrench space origin then the grasp is stable. One quality measure that is often proposed is the radius, $\epsilon$, of the largest $6 \mathrm{D}$ ball, centered at the origin, that can be enclosed with the hull. The vector from the wrench space origin to the point where the ball contacts the boundary of the hull is the smallest maximum wrench that can be applied by the grasp. In this worst case the sum magnitude of the contact wrenches would have to be $\frac{1}{\epsilon}$ times the magnitude of the disturbance wrench. The closer $\epsilon$ is to 1 the more efficient the grasp is. However, this measure is not invariant to the choice of torque origin as $\mathrm{Li}$ and Sastry point out, so the volume $v$ of the convex hull can be used as an invariant average case quality measure for the grasp.

We have implemented this portion of the analysis with the Qhull program [2]. It produces a list of facets of the convex hull, and each is described by a normal 
vector and a signed offset from the origin. This makes it easy to determine whether the origin is contained in the hull, indicating a stable grasp, and which facet is closest to the origin, indicating the most difficult wrench for the grasp to apply. Clearly, the wrench in the opposite direction is the most difficult external wrench for the grasp to resist. The minimum offset value gives us $\epsilon$ and indicates how efficient the grasp is at handling this worst case. Qhull can also be queried for the volume of the computed hull which gives us $v$.

\subsection{D Hull Projections}

One reason $3 \mathrm{D}$ examples are avoided in previous papers is due to the difficulty of visualizing the results. A 2D planar object will have a 3D wrench space, but a $3 \mathrm{D}$ object will have a $6 \mathrm{D}$ wrench space, and to display it, we must project it into $3 \mathrm{D}$ space by fixing three of the wrench coordinates. We have chosen four projections of the grasp wrench space that can help us understand some of the characteristics of a particular grasp. If we fix the torque coordinates of the wrench space to 0 , the resulting projection shows the space of forces that can be applied by the grasp without imparting a net torque to it, and likewise, if we fix the force coordinates to 0 , we can visualize the space of torques that can be applied to the object without a net force acting on the body. By setting the torque coordinates to their values at the point on the hull boundary that is closest to the origin, $\mathbf{w}_{\min }^{\tau}$, we can display the space of forces that can be applied if the worst case torque needs to be applied. Similarly we can display the space of torques that can be applied if the worst case force, $\mathbf{w}_{\min }^{f}$, must be applied. In general, we start with the collection of $6 \mathrm{D}$ halfspaces, $H_{n \times 6} \mathbf{x} \leq \mathbf{b}$, but once we have chosen values for three coordinates of $x$, we arrive at a new set of $3 \mathrm{D}$ halfspaces, $H_{n \times 3}^{\prime} \mathbf{x} \leq \mathbf{b}^{\prime}$. From there we use Qhull again to compute their intersection which gives us the hull bodies that are presented in the next section.

\section{Results}

In this section, we present several examples of different grasps of a telephone handset performed using models of two different actual robotic hands. Each section begins with a description of the hand used and continues with a demonstration of the process described in the previous section. We show every step taken by the simulator, and we display selected projections of the resulting convex hulls that help to visualize some of the properties of each grasp. These examples are unique because they are the first to illustrate the quality computations for $3 \mathrm{D}$ grasps; whereas previously, others have only provided the results of $2 \mathrm{D}$ grasp quality computations. It is important to note not only the system's ability to handle 3D grasps, but also its ability to locate contacts with a fine level of detail and to handle these contacts even if they are complex, or involve friction or curved surfaces.

\subsection{The Barrett Hand}

The dextrous robot hand used for this portion of our investigation is the Barrett Hand [1]. It is an eightaxis, three-fingered mechanical hand with each finger having two joints. One finger is stationary and the other two can spread synchronously up to 180 degrees about the palm (finger 3 is stationary and fingers 1 and 2 rotate about the palm). Although there are eight axes, the hand is controlled by four motors. Each of the three fingers has one actuated proximal link, and a coupled distal link that moves at a fixed rate with the proximal link. A novel clutch mechanism allows the distal link to continue to move if the proximal link's motion is obstructed (referred to as breakaway). An additional motor controls the synchronous spread of the two fingers about the palm.

The Barrett hand has 10 degrees of freedom: 6 for the pose of the wrist, 1 for the spread angle of the fingers, and 3 for the angles of the proximal links. However, these last 3 do not need to be specified in the hand's initial configuration with respect to the object because the fingers will simply close from their fully open position until contact is made. Our current model of the hand uses slightly simplified link geometries, but the kinematics of the model match the real hand exactly. Because the actual hand in our lab is outfitted with tactile sensors on the inner pad of each link, including the palm, we have set the link material of each link in the model to rubber. Our object to be grasped is a telephone handset, and in an object configuration file, we describe it with hand-generated polyhedral model and specify its material as plastic.

Our first grasp with this hand (grasp 1), positions the hand with its palm flat against a side surface of the phone and the fingers wrapping completely around (see figure 2). While many researchers do not take the palm into consideration, we have found that it can greatly aid grasp stability. Next, we show the friction cones that have been placed on the vertices of contact regions. The proximal link of finger 1 (colored light red) ${ }^{1}$ has a point-plane contact which causes the distal link (dark red) to breakaway and continue to close, resulting in a line-plane contact as the edge of the finger contacts the side face of the phone. The distal links of fingers 2 and 3 (dark blue and dark green) have

\footnotetext{
${ }^{1} \mathrm{~A}$ color version of this paper is available at http://www.cs.columbia.edu/ amiller
} 

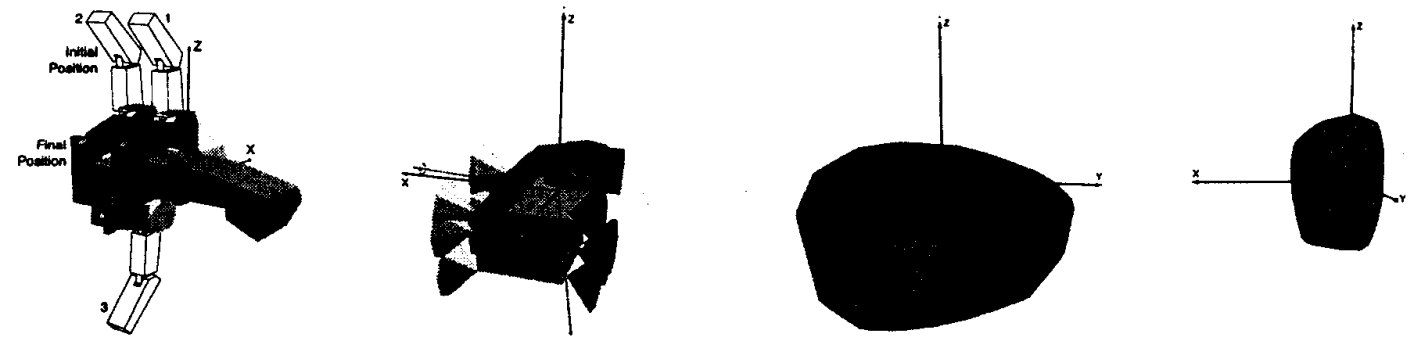

Figure 2: (1): Initial and final configurations of the Barrett hand for example grasp 1. The simulator found the values for the inner joints which result in contact. (2): Friction cones placed at the vertices of contact regions. [Note in all figures depicting the handset, axes are drawn through its center of gravity.] (3): Hull 1 projected into force space with $\tau=w_{m i n}^{\tau}$. The maximal sphere is restricted by a facet above it. (4): Hull 1 projected into torque space with $f=w_{\min }^{f}$. The maximal sphere is restricted by a facet with a normal close to the $-x$ axis.

line-line contacts as a side edge of each link touches a sloping edge along the side of the phone. The normals of these contacts are determined by the cross product of the two contacting edges, and therefore, they are not quite perpendicular to the face of the phone. Finally, there is a large plane-plane contact between the palm (yellow) and the other side face of the phone, and one friction cone is placed at each vertex of the shared contact region. This figure also indicates the direction of the force and torque components of the most difficult wrench for the grasp to resist. As described earlier, this wrench is in the opposite direction as the normal of the $6 \mathrm{D}$ hull facet that is closest to the wrench space origin. Currently, we only show the direction of these components due to the difficulty of displaying their relative magnitudes.

To understand how we arrived at these components, we also show the force and torque space projections of the computed $6 \mathrm{D}$ grasp wrench space. At the origin of each of these spaces, is a projection of the maximal $6 \mathrm{D}$ ball that just fits within the grasp wrench space. One can see from the force hull, which assumes we wish to use these points of contact to resist the torque component of the worst case wrench (roughly about the $x$ axis), it is very difficult to also apply a force in the positive $z$ direction. The torque hull is a projection that assumes we are trying to resist the force component of worst case wrench (roughly along the $-z$ axis), and we can see that the most difficult torques to apply in this case are about the $-x$ axis.

Figure 3 shows a grasp that our system classified as unstable. While the grasp would be sufficient to lift the phone, it is not a form closure grasp. The convex hull of the contact wrenches does not include the wrench space origin because it does not include any wrenches with a force component in the $-z$ direction. Visually, one can verify that the grasp cannot resist upward pushing forces.
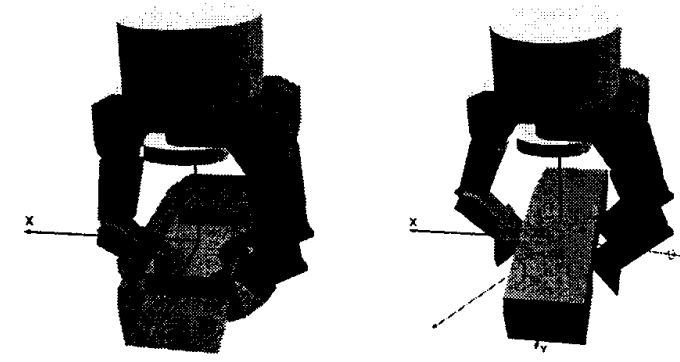

Figure 3: Left: Unstable grip. Right: Example grasp 2.

Next we present a grasp from slightly farther above the phone. There are now three line contacts between the edges of each of the outer links and the side faces of the phone. In this example, we chose to project the convex hull into force and torque space by fixing the torque and force components to zero (figure 4). These hulls show us which forces can be applied by the grasp without applying a net torque or vice versa. Note the symmetry in the hulls due to the symmetrical placement of the contacts about the center of gravity. The force hull shows us that a wide variety of large forces can be applied without a net torque because of the balanced contacts and the large coefficient of friction at all of the contacts. In addition, it is obvious from the size of the torque hull that this grasp cannot apply large pure torques on the phone because of the proximity of the contacts to the origin.
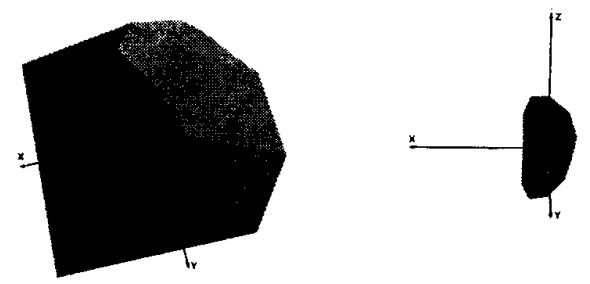

Figure 4: Hull 2 projected into force space with $\tau=0$ (left) and into torque space with $f=0$ (right). 


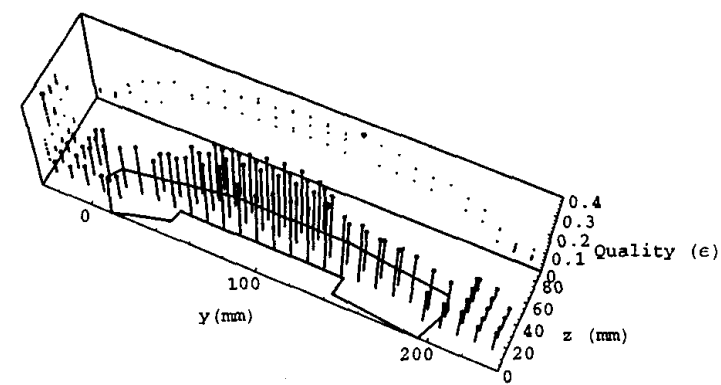

Figure 5: Quality surface. The highest quality position is marked with the larger ball.

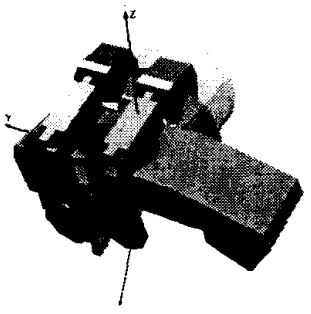

Figure 6: Example grasp 3.

\subsubsection{Searching for High Quality Grasps}

The position of our third example grasp was actually chosen from a search for optimal quality over a small subspace of our configuration parameters. The first grasp showed us that the palm contact is greatly beneficial to the overall quality of the grasp. We fixed the orientation and the $x$ parameter of the hand's translation such that the palm was flat against the side of the phone as in the first grasp. We also fixed the finger spread angle at 0 degrees, leaving us two degrees of freedom for us to vary: the $y$ and $z$ translation of the hand. The center of the palm was moved along a grid of sample points at $15 \mathrm{~mm}$ increments, and using a preprocessing step to examine at each position whether all of the links were clear of the phone, we created a list of 82 valid positions for the hand. The simulator then examined each of those positions and recorded the quality for that grasp. The running time for the search was 1 hour and 28 minutes, and approximately $90 \%$ of that time was spent locating contacts. Figure 5 is a graph of the quality at each of these sample points along one face of the phone, and figure 6 shows the highest quality grasp found in this search.

\subsection{The DLR Hand}

The DLR hand [3], developed at the German Aerospace Center, is a four-fingered articulated robotic hand, and it has an anthropomorphic design. The fingers are identical, and each consists of three links with two joints at the base, one joint between the proximal and medial links, and one joint between the medial and distal links. This last joint is coupled in a fixed ratio to the previous joint in the chain just as it is in the human hand. The DLR hand has a total of 18 degrees of freedom: 6 specify the pose of the wrist, and there are 3 independently controllable joints in each of the 4 fingers.

In the description file for the DLR hand, we specify its kinematics, including joint limits, and its link materials (metal for the palm, proximal, and medial links, rubber for distal links). Also specified are the link models, which are accurate replicas of the actual hand, except we have omitted unnecessary details at the joints. To begin the analysis of a grasp, we set the wrist pose with respect to the phone, and we fix the values of the two base joints in each finger. However, we leave the joint between the proximal and medial links and the coupled joint between the medial and distal links of each finger free to move. The simulator then analyzes the grasp that will result at this configuration in the same manner as before (see figure 7).

The right portion of this figure shows the friction cones that have been added at each contact. Because the palm of the DLR hand is curved, it can only touch the phone along two line contacts in this configuration. Looking closely, we see that these are actually narrow planar surface contacts (line contacts should only have one cone at each endpoint). This occurs because the palm model provided to us by DLR is faceted, but this approximation has a negligible effect on the quality of the grasp. Each rounded finger tip contacts the other side of the phone at individual points. The small size of the friction cones along the palm contacts reflects the low coefficient of friction for a metal-plastic contact, but the larger friction cones at the fingertip contacts reflect the strength of rubber-plastic contacts. Again, this figure also indicates the components of the worst case disturbance wrench.

To verify these directions, we show the $3 \mathrm{D}$ force and torque hull projections (figure 8 ). As expected, the normals of the restricting facets in each hull and the
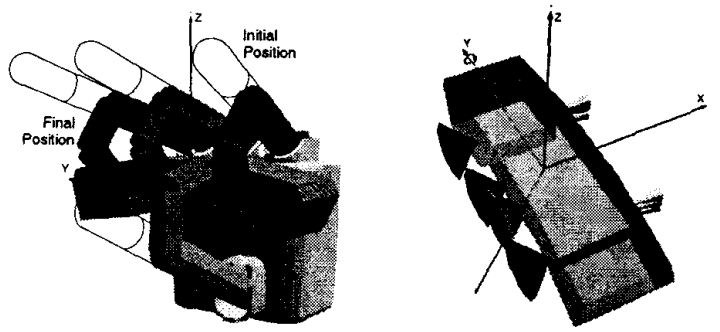

Figure 7: Left: The initial and final configuration of the DLR hand for example grasp 4. Right: Friction cones placed at the vertices of the contact regions. 


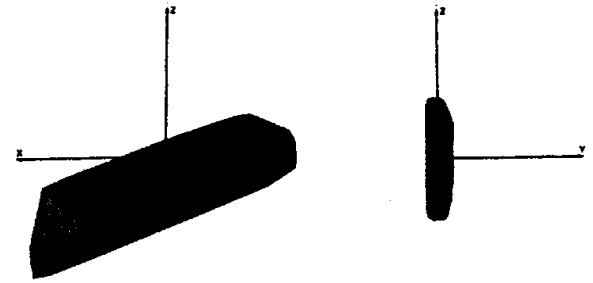

Figure 8: Left: Hull 4 projected into force space with $\tau=\mathbf{w}_{\text {min }}^{\tau}$. The tiny maximal sphere located at the origin is restricted by a hull facet that lies very close to the origin and has an approximate normal of $(0.4,0,0.9)$. Right: Hull 4 projected into torque space with $f=w_{\min }^{f}$. A facet with a normal along the $-y$ axis restricts the maximal sphere.

components of the worst case wrench are in opposite directions. Because the contact points are distributed along the $y$ axis and lie close to the $x y$ plane, it is very difficult to resist torques about the $y$ axis. Furthermore, since there are no contacts on the top or bottom surfaces of the phone, it is difficult to resist forces applied in vertical directions. Thus, the results of this grasp analysis match what our common sense tells us about this grasp.

\begin{tabular}{||c|c|c|c||}
\hline Grasp & Hand & Quality $\epsilon$ & Quality $v$ \\
\hline \hline 1 & Barrett & 0.258 & 0.849 \\
\hline 2 & Barrett & 0.197 & 0.113 \\
\hline 3 & Barrett & 0.335 & 1.09 \\
\hline 4 & DLR & 0.0966 & 0.0783 \\
\hline
\end{tabular}

Table 1: Grasp quality of example grasps.

\section{Conclusion and Future Work}

We have demonstrated a system that, given models of a hand and an object, can compute two measures of grasp quality and allow us to visualize the results of these computations.

Table 1 shows the computed quality values for each of the example grasps. Both quality measures rank the grasps the same with the third grasp as our best candidate examined so far. Note that these measures compare the stability of a grasp only, and alone they do not provide a fair comparison between the hands since the Barrett hand was designed to create power grasps, while the DLR hand was designed for in-hand manipulation.

Future work will include the construction of a grasping library consisting of a variety of hand and object models, as well as additional grasp quality measures. This library will allow us to perform many comparative tests and help answer the question of how the kinematic and geometric design of a hand affects grasp stability and grasp manipulability. In the area of grasp synthesis, this paper presents our initial work in locating an optimal grasp of an object, and while the total space of hand configurations is prohibitively large, we will be improving the speed of the contact location phase of our system and attempting to synthesize locally optimal grasps from a given starting point.

Acknowledgment: We would like to thank Professor Gerd Hirzinger and Dr. Max Fischer from the German Aerospace Center (DLR) for providing us with models of their robotic hand.

\section{References}

[1] P. K. Allen, A. T. Miller, P. Y. Oh, and B. S. Leibowitz. Using tactile and visual sensing with a robotic hand. In Proc. of the 1997 IEEE Int. Conf. on Robotics and Automation, April 1997.

[2] C. B. Barber, D. P. Dobkin, and H. Huhdanpaa. The Quickhull algorithm for convex hulls. ACM Transactions on Mathematical Software, 22(4):469-483, December 1996.

[3] J. Butterfass, G. Hirzinger, S. Knoch, and H. Liu. DLR's multisensory articulated hand, part I: Hard- and software architecture. In Proc. of the 1998 IEEE Int. Conf. on Robotics and Automation, pages 2081-2086, 1998.

[4] C. Ferrari and J. Canny. Planning optimal grasps. In Proc. of the 1992 IEEE Int. Conf, on Robotics and Automation, pages 2290-2295, 1992.

[5] M. Fischer and G. Hirzinger. Fast planning of precision grasps for 3D objects. In Proc. of the 1997 IEEE/RSJ Int. Conf. on Intelligent Robots $\mathcal{B}$ Systems, pages 120-126.

[6] D. Kirkpatrick, B. Mishra, and C. K. Yap. Quantitative Steinitz's theorems with applications to multifingered grasping. In Proc. of the 20th ACM Symp. on Theory of Computing, pages 341-351, 1990.

[7] Z. Li and S. Sastry. Task-oriented optimal grasping by multifingered robot hands. IEEE Journal of Robotics and Automation, RA-4(1):32-44, February 1988.

[8] B. Mishra and N. Silver. Some discussion of static gripping and its stability. IEEE Journal on Systems, Man, and Cybernetics, 19(4):783-796, August 1989.

[9] V.-D. Nguyen. Constructing force-closure grasps. In Proc. of the 1986 IEEE Int. Conf. on Robotics and Automation, pages 2290-2295, 1986.

[10] J. Pertin-Troccaz. Grasping: A state of the art. In O. Khatib, J. J. Craig, and T. Lozano-Pérez, editors, The Robotic Review, volume 1, pages 71-98. MIT Press, Cambridge, MA, 1989.

[11] N. S. Pollard. Parallel Methods for Synthesizing WholeHand Grasps from Generalized Prototypes. PhD thesis, Department of Electrical Engineering and Computer Science, MIT, 1994

[12] J. K. Salisbury. Kinematic and Force Analysis of Articulated Hands. PhD thesis, Department of Mechanical Engineering, Stanford University, 1982. 